\title{
First stars $X$. The nature of three unevolved carbon-enhanced metal-poor stars ${ }^{\star}$
}

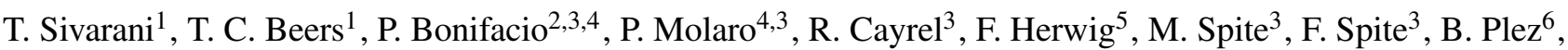 \\ J. Andersen ${ }^{7,8}$, B. Barbuy ${ }^{9}$, E. Depagne ${ }^{10}$, V. Hill ${ }^{3}$, P. François ${ }^{3}$, B. Nordström ${ }^{11,7}$, and F. Primas ${ }^{12}$ \\ ${ }^{1}$ Department of Physics \& Astronomy, CSCE: Center for the Study of Cosmic Evolution, and JINA: Joint Institute for Nuclear \\ Astrophysics, Michigan State University, East Lansing, MI 48824, USA \\ e-mail: thirupathi@pa.msu.edu \\ 2 CIFIST Marie Curie Excellence Team \\ 3 Observatoire de Paris, GEPI, 92195 Meudon Cedex, France \\ ${ }^{4}$ Istituto Nazionale di Astrofisica - Osservatorio Astronomico di Trieste, via Tiepolo 11, 34131 Trieste, Italy \\ 5 Los Alamos National Laboratory (LANL), Los Alamos, New Mexico, NM 87545, USA \\ ${ }^{6}$ GRAAL, Université de Montpellier II, 34095 Montpellier Cedex 05, France \\ 7 The Niels Bohr Institute, Astronomy, Juliane Maries Vej 30, 2100 Copenhagen, Denmark \\ 8 Nordic Optical Telescope Scientific Association, Apartado 474, 38700 Santa Cruz de La Palma, Spain \\ 9 Universidade de Sao Paulo, Departamento de Astronomia, Rua do Matao 1226, 05508-900 Sao Paulo, Brazil \\ 10 European Southern Observatory (ESO), 3107 Alonso de Cordova, Vitacura, Casilla 19001, Santiago 19, Chile \\ 11 Lund Observatory, Box 43, 22100 Lund, Sweden \\ 12 European Southern Observatory (ESO), Karl-Schwarschild-Str. 2, 85749 Garching b. München, Germany
}

Received 14 April 2006 / Accepted 8 July 2006

\section{ABSTRACT}

\begin{abstract}
Context. On the order of $20 \%$ of the very metal-poor stars in the Galaxy exhibit large carbon enhancements. It is important to establish which astrophysical sites and processes are responsible for the elemental abundance patterns of this early Galactic population. Aims. We seek to understand the nature of the progenitors of three main-sequence turnoff Carbon-Enhanced Metal-Poor (CEMP) stars, CS 31080-095, CS 22958-042, and CS 29528-041, based on a detailed abundance analysis.

Methods. From high-resolution VLT/UVES spectra $(R \sim 43000)$, we determine abundances or upper limits for Li, C, N, O, and other important elements, as well as ${ }^{12} \mathrm{C} /{ }^{13} \mathrm{C}$ isotopic ratios.

Results. All three stars have $-3.30 \leq[\mathrm{Fe} / \mathrm{H}] \leq-2.85$ and moderate to high CNO abundances. CS $22958-042$ is one of the most carbon-rich CEMP stars known $([\mathrm{C} / \mathrm{Fe}]=+3.2)$, while CS 29528-041 (one of the few N-enhanced metal-poor stars known) is one of the most nitrogen rich $([\mathrm{N} / \mathrm{Fe}]=+3.0)$. Oxygen is very high in CS 31080-095 $([\mathrm{O} / \mathrm{Fe}]=+2.35)$ and in CS $22958-042$ $([\mathrm{O} / \mathrm{Fe}]=+1.35)$. All three stars exhibit $[\mathrm{Sr} / \mathrm{Fe}]<0$; $\mathrm{Ba}$ is not detected in $\mathrm{CS} 22958-042([\mathrm{Ba} / \mathrm{Fe}]<-0.53)$, but it is moderately enhanced $([\mathrm{Ba} / \mathrm{Fe}] \sim 1)$ in the other two stars. CS 22958-042 displays one of the largest sodium overabundances yet found in CEMP stars $([\mathrm{Na} / \mathrm{Fe}]=+2.8)$. CS $22958-042$ has ${ }^{12} \mathrm{C} /{ }^{13} \mathrm{C}=9$, similar to most other CEMP stars without enhanced neutron-capture elements, while ${ }^{12} \mathrm{C} /{ }^{13} \mathrm{C} \geq 40$ in CS 31080-095. CS 31080-095 and CS 29528-041 have $A(\mathrm{Li}) \sim 1.7$, below the Spite Plateau, while $\mathrm{Li}$ is not detected in CS 22958-042.

Conclusions. CS 22958-042 is a CEMP-no star, but the other two stars are in no known class of CEMP star and thus either constitute a new class or are a link between the CEMP-no and CEMP-s classes, adding complexity to the abundance patterns for CEMP stars. We interpret the abundance patterns in our stars to imply that current models for the presumed AGB binary progenitors lack an extra-mixing process, similar to those apparently operating in RGB stars.
\end{abstract}

Key words. stars: abundances - stars: population II - Galaxy: abundances - stars: AGB and post-AGB

\section{Introduction}

A considerable fraction $(20-25 \%)$ of the very metal-poor stars in the Galaxy $([\mathrm{Fe} / \mathrm{H}] \leq-2.0)$ are strongly enhanced in carbon and often in nitrogen as well $([\mathrm{C}, \mathrm{N} / \mathrm{Fe}]>1.0$; Beers \& Christlieb 2005; Lucatello et al. 2006). It appears that multiple nucleosynthetic pathways for the production of $\mathrm{C}$ in low-metallicity stars must be invoked, almost certainly involving different astrophysical sites. Most stars in the range $-2.6 \leq[\mathrm{Fe} / \mathrm{H}] \leq-2.0$ appear to be associated with $\mathrm{C}$ production in Asymptotic Giant Branch

* Based on observations made with the ESO Very Large Telescope at Paranal Observatory, Chile (Large programme "First Stars", ID 165.N0276(A); P.I.: R. Cayrel).
(AGB) companions that have transferred material to the star that is now observed (Aoki et al. 2006a). Even lower-metallicity stars, especially those with $[\mathrm{Fe} / \mathrm{H}]<-3.0$, may contain $\mathrm{C}$ produced by massive, rapidly-rotating stars with $[\mathrm{Fe} / \mathrm{H}]<-6.0$; see, e.g., Hirschi et al. (2006), Karlsson et al. (2006), Piau et al. (2006). In order to better understand the role of these various possible mechanisms, detailed investigations are needed of the elemental abundances in Carbon-Enhanced Metal-Poor (CEMP) stars $([\mathrm{C} / \mathrm{Fe}]>+1.0$; see Beers \& Christlieb 2005).

Recent high-resolution spectroscopic studies of CEMP stars selected from the two large objective-prism surveys for lowmetallicity stars, the HK (Beers et al. 1985; Beers et al. 1992; Beers 1999) and Hamburg-ESO surveys (Christlieb 2003), have revealed a wide variety of elemental abundance patterns 
(e.g., Norris et al. 1997b,c; Bonifacio et al. 1998; Aoki et al. 2002a,b,c; Barklem et al. 2005; Beers \& Christlieb 2005). Many of these stars (roughly 70-80\%; Aoki et al. 2003, 2006a) exhibit strong s-process enhancements (CEMP-s), and are thought to be the result of AGB mass transfer to the presently observed companion (e.g., Aoki et al. 2002c; Lucatello et al. 2003; Sivarani et al. 2004; Barbuy et al. 2005).

The observed radial-velocity variations among the CEMP-s stars indicate that most, if not all, could be members of binary systems (Tsangarides et al. 2004; Lucatello et al. 2005). Even within this group of CEMP-s stars, the observed s-process patterns are not identical, however. For example, the scatter in their observed $\mathrm{Sr}, \mathrm{Ba}, \mathrm{Pb}$, and $\mathrm{C}$ abundances at a given $[\mathrm{Fe} / \mathrm{H}]$ is large (e.g., Aoki et al. 2002c; Sivarani et al. 2004; Barbuy et al. 2005). This diversity presumably arises because of the range in masses of the star that underwent AGB evolution (see Herwig 2005 for a recent review), as well as due to details of internal mixing and possible variations in the level of dilution of the material passed to the star we now observe.

Other CEMP stars exhibit r-process enhancements (e.g., CS 22892-052; Sneden et al. 2003); Lucatello (private communication) reports several additional likely CEMP-r stars from an analysis of the HERES sample of Barklem et al. (2005). Another class of carbon-enhanced metal-poor stars are the CEMP-r/s stars, which exhibit the presence of neutron-capture elements associated with both the r- and s-process (e.g., CS 22948-027, CS 29497-034, Hill et al. 2000 and Barbuy et al. 2005; CS 29526-110, CS 22898-027, CS 31062-012, Aoki et al. 2002c; HE 2148-1247, Cohen et al. 2003; CS 29497-030, Ivans et al. 2005).

Most CEMP-r/s stars also exhibit very high $\mathrm{Pb}$ abundances (the so-called "lead stars," see Aoki et al. 2002c; and van Eck et al. 2003). The discovery of CS 22957-027 (Norris et al. 1997c; Bonifacio et al. 1998) and a number of similar stars which exhibit no enhancements of neutron-capture elements (Aoki et al. 2002a,b; Depagne et al. 2002; Plez \& Cohen 2005; Frebel et al. 2005), led Beers \& Christlieb (2005) to introduce the CEMP-no class of stars.

As shown below, at least one of the three stars studied here is classified as a CEMP-no star. The other two stars in our study exhibit intermediate $\mathrm{Ba}$ excesses $([\mathrm{Ba} / \mathrm{Fe}]>0)$, but no other strong s-process-element enrichment. Thus, they fail the Beers \& Christlieb (2005) criterion for the Ba ratios in CEMP-no stars (which requires $[\mathrm{Ba} / \mathrm{Fe}]<0.0$ ), but they may well be related to this class.

The origin of CEMP-no stars remains unclear at present. They could, for example, be the result of pollution by AGB stars which, for some reason, have been unable to activate the sprocess, but have still dredged up significant amounts of carbon to their outer atmospheres. Alternatively, the high CNO abundances in these stars could be the products of SNe; such models have been proposed at least in the case of HE 0107-5240 (Christlieb et al. 2002; Bonifacio et al. 2003) and HE 1327-2326 (Frebel et al. 2005; Iwamoto et al. 2005).

Measurements of $\mathrm{C}, \mathrm{N}$, and $\mathrm{O}$ abundances provide crucial information on the nucleosynthetic and mixing history of the progenitors of CEMP stars. The ${ }^{12} \mathrm{C} /{ }^{13} \mathrm{C}$ ratio is a sensitive indicator of the mixing processes experienced by carbon-enhanced stars; $\mathrm{Li}$ provides another valuable diagnostic tool. Lithium is not detected in most CEMP stars, but is sometimes found at a level below the Spite Plateau (Norris et al. 1997a; Aoki et al. 2002b); both observations suggest that some mechanism induced strong mixing and Li burning in the past. However, at least two known CEMP stars, LP 706-7 (Norris et al. 1997b) and CS 22898-027
(Thorburn \& Beers 1992) exhibit Li abundances corresponding to the Spite Plateau. Perhaps surprisingly, both of these stars are neutron-capture-element-rich stars (Norris et al. 1997b; Aoki et al. 2002c), although significant radial-velocity variation has yet to be found in either star.

In this paper we present a detailed abundance analysis for three main-sequence turnoff CEMP stars, CS 31080-095, CS 22958-042, and CS 29528-041, based on high-resolution, high $S / N$ observations obtained with the ESO VLT/UVES. Main-sequence turnoff CEMP stars are of particular interest because their present evolutionary stage precludes the significant internal mixing that is expected for cooler CEMP stars, hence their atmospheres better preserve the nucleosynthetic signatures of their progenitors.

We describe our observations in Sect. 2. Details of our abundance analysis procedures are reported in Sect. 3; in Sect. 4 we present the results, and in Sect. 5 we examine possible scenarios for the nucleosynthesis histories of these objects and compare them with the properties of previously observed CEMP stars. Our conclusions are presented in Sect. 6.

Throughout this paper, the abbreviations VMP, EMP, UMP, HMP, and MMP (Very, Extremely, Ultra, Hyper, and Mega Metal Poor) denote stars with $[\mathrm{Fe} / \mathrm{H}]$ below $-2.0,-3.0,-4.0$, -5.0 , and -6.0 , respectively (Beers \& Christlieb 2005).

\section{Observations and data reduction}

Our spectroscopic data were obtained at the VLT-Kuyen $8.2 \mathrm{~m}$ telescope and UVES spectrograph (Dekker et al. 2000) at a resolving power of $R=43000$. The coordinates of our program stars and the log of observations are listed in Table 1. Non-standard grating settings were used, both with dichroic \#1: $396+573,396+850$ (central wavelengths in $\mathrm{nm}$ in the blue and red arm, respectively). The central wavelengths in the red arm were chosen so that both settings would cover the Li doublet, effectively doubling the integration time on this important line.

The data were reduced using the UVES context within MIDAS, which includes bias subtraction, flat fielding, wavelength calibration, and the merging of echelle orders. The continuum normalisation was performed with IRAF (using a cubic spline function) for the merged spectra. For a few lines that were either very weak, or of particular interest, we have used the spectra of the individual orders without merging them. Balmer-line profiles were checked using both single-order and merged spectra. Equivalent width measurements for unblended lines were obtained by fitting Gaussian profiles, using the genetic algorithm code described by François et al. (2003).

Radial velocities (listed in Table 1) were measured from unblended lines in the range 380-450 nm. Multiple-epoch observations of CS 31080-095 (over a range of 386 days) and of CS 29528-041 (over a range of 45 days) do not reveal any detectable radial-velocity variations, while quite large $\left(3 \mathrm{~km} \mathrm{~s}^{-1}\right)$ variations are found in CS 22958-042 over only one hour. If this velocity variation is correct, CS 22958-042 may be another example of a very short-period binary similar to HE 0024-2523, as reported previously by Lucatello et al. (2003).

$U B V$ photometry for CS $29528-041$ is available from Norris et al. (1999). For the other two stars, we obtained broadband photometry with the Danish $1.5 \mathrm{~m}$ telescope at ESO, Chile (Beers et al., in preparation). Near infrared $J H K$ photometry for all three stars was available from the 2MASS catalog (Skrutskie et al. 2006). Estimates of interstellar reddening along the line of sight to each star were obtained from Schlegel et al. (1998). We also adopt Table 6 from Schlege et al. (1998) for the relative 
Table 1. Log of observations.

\begin{tabular}{lclllll}
\hline \hline Star & $\begin{array}{c}\text { Coordinates } \\
(2000)\end{array}$ & $\begin{array}{l}\text { Date } \\
\mathrm{y} / \mathrm{m} / \mathrm{d}\end{array}$ & MJD-24000.5 & $\begin{array}{l}\text { Exposure } \\
(\mathrm{s})\end{array}$ & $\begin{array}{l}\text { Radial velocity } \\
\mathrm{km} \mathrm{s}^{-1}\end{array}$ & $\begin{array}{l}S / N \\
\text { at } 430 \mathrm{~nm}\end{array}$ \\
\hline CS 31080-095 & $04: 44: 21.8-45: 13: 57$ & $2000-10-19$ & 51836.2572450 & 2400 & -43.64 & 93 \\
& & $2000-10-19$ & 51836.2864673 & 2400 & -43.30 & 72 \\
& & $2001-10-19$ & 52201.3331806 & 3600 & -43.76 & 82 \\
& & $2001-10-20$ & 52202.3388291 & 3000 & -43.76 & 52 \\
& & $2001-11-06$ & 52219.2454194 & 3600 & -43.66 & 92 \\
CS 22958-042 & $02001-11-09$ & 52222.2693614 & 1000 & -43.34 & 48 \\
& & & & & \\
CS 29528-041 & $02: 01: 07.5-57: 17: 07$ & $2001-11-05$ & 52218.1900217 & 3600 & +165.21 & 48 \\
& & $2001-11-05$ & 52218.2360571 & 3600 & +162.51 & 35 \\
& & & & & & 50 \\
& & & & & -285.1 & 33 \\
\end{tabular}

Table 2. Photometry and adopted atmospheric parameters.

\begin{tabular}{lrrr}
\hline \hline Parameter & CS 31080-095 & CS 22958-042 & CS 29528-041 \\
\hline$V$ & 12.989 & 14.516 & 14.60 \\
$B-V$ & 0.521 & 0.479 & 0.42 \\
$U-B$ & -0.291 & $\ldots$ & -0.19 \\
$V-R$ & 0.317 & 0.294 & $\ldots$ \\
$V-I$ & 0.617 & 0.614 & $\ldots$ \\
$V-K$ & 1.397 & 1.303 & 1.292 \\
$J-H$ & 0.272 & 0.227 & 0.314 \\
$J-K$ & 0.350 & 0.302 & 0.339 \\
& & & \\
$E(B-V)$ & 0.009 & 0.025 & 0.030 \\
& & & \\
$T_{\text {eff }}(B-V)_{0}$ & 5671 & 5897 & 6378 \\
$T_{\text {eff }}(V-R)_{0}$ & 5964 & 6221 & $\ldots$ \\
$T_{\text {eff }}(V-I)_{0}$ & 6020 & 6146 & $\ldots$ \\
$T_{\text {eff }}(R-I)_{0}$ & 6224 & 6135 & $\ldots$ \\
$T_{\text {eff }}(V-K)_{0}$ & 6077 & 6344 & 6199 \\
$T_{\text {eff }}(J-H)_{0}$ & 5940 & 6277 & 5947 \\
$T_{\text {eff }}(J-K)_{0}$ & 5822 & 6156 & 6266 \\
Adopted: & & & \\
$T_{\text {eff }}($ Fe lines $)$ & 6050 & 6250 & 6150 \\
$\log g$ & 4.50 & 3.50 & 4.00 \\
{$[$ Fe $/ \mathrm{H}]$} & -2.85 & -2.85 & -3.30 \\
$\xi_{\mathrm{t}}\left(\mathrm{km} \mathrm{s} \mathrm{s}^{-1}\right)$ & 1.0 & 1.5 & 1.3 \\
\hline
\end{tabular}

extinctions of various bandpasses. The photometric data and derived parameters are listed in Table 2.

All our program stars have intermediate-band Strömgren photometry by Schuster et al. (2005), who estimated metallicities and photometric classifications as follows: CS 31080-095: MS (CH), CS 22958-042: TO (CH), and CS 29528-041: TO, respectively; these classifications are consistent with our derived surface gravities, as described below.

\section{Analysis}

For a first estimate of the $T_{\text {eff }}$ for our program stars, we employed the various colours with the Alonso et al. (1996) calibrations of $T_{\text {eff }}$ for dwarf stars. The results are listed in Table 2. Transformations between the different photometric systems necessary for use of the Alonso et al. calibrations were carried out as described in Sivarani et al. (2004). With the exception of temperatures based on the $B-V$ colour, which is susceptible to the effect of molecular carbon absorption on the $B$-band flux, the colour-based estimates of $T_{\text {eff }}$ agree to within $125-200 \mathrm{~K}$.

As a likely better approach to estimating $T_{\text {eff }}$ we have also used the Fe I excitation equilibrium. The effective temperatures derived from this procedure, also listed in Table 2, fall within $100 \mathrm{~K}$ of the average of the colour-based estimates of $T_{\text {eff }}$ (not including the estimate based on $B-V$ ). In particular, they agree very well (within $50 \mathrm{~K}$ ) with the temperature from $V-K$, which should be superior due to the large baseline and the fact that both the $V$ and $K$ bands are relatively free of molecular carbon features. We adopt the spectroscopic $T_{\text {eff }}$ estimates for the remainder of our analysis.

The surface gravity, $\log g$, was derived from the $\mathrm{Fe}$ I/Fe II ionisation equilibrium, and the microturbulence was determined by requiring that the abundances from the $\mathrm{Fe}$ I lines be independent of equivalent width. The gravity we obtain for CS 31080-095 $(\log g=4.50)$ is consistent with the dwarf classification by Schuster et al. (2005), and our results for CS 22958-042 $(\log g=3.50)$ and CS 29528-041 $(\log g=4.00)$ also correspond to main-sequence TO stars. The slightly lower gravity of CS 22958-042 may indicate that it is a subgiant that has evolved slightly away from the turnoff.

\section{Abundances}

We employ the same model atmospheres (OSMARCS; see Gustafsson et al. 2003 and references therein, with appropriate $\mathrm{C}$ and $\mathrm{N}$ enhancement), current version of the spectrum synthesis code (turbospectrum; Alvarez \& Plez 1998), and line lists as in previous papers of this series (Hill et al. 2000; Depagne et al. 2002; François et al. 2003). We also use the $\mathrm{CH}$ molecular line list compiled by Plez (Plez \& Cohen 2005). The NH and $\mathrm{C}_{2}$ molecular linelists are taken from the Kurucz database (http://kurucz . harvard. edu/linelists/linesmol/).

Our derived elemental abundances are listed in Table 3. Errors in $\log (\epsilon)$ are assigned for stars with multiple lines, based on the errors in the mean (i.e., $\sigma(\log (\epsilon)) / \sqrt{(N)})$; no errors are listed for elements in which only one line was measured. Total errors in $[\mathrm{X} / \mathrm{Fe}]$ for each element are of the order of $0.10-0.15 \mathrm{dex}$, taking our adopted errors of $100 \mathrm{~K}$ in $T_{\text {eff }}$ and $0.5 \mathrm{dex}$ in $\log g$ into account. We adopt the solar abundances of Asplund et al. (2005).

CS 22958-042 has no detectable $\mathrm{Ba}([\mathrm{Ba} / \mathrm{Fe}]<-0.53)$ (Table 3); it is thus classified as a CEMP-no star, according to Beers \& Christlieb (2005). The other two stars exhibit moderate enhancements of $\mathrm{Ba}([\mathrm{Ba} / \mathrm{Fe}]=+0.77$ and +0.97$)$. These 
Table 3. Elemental abundance and isotopic ratio measurements for three CEMP stars.

\begin{tabular}{|c|c|c|c|c|c|c|c|}
\hline \multirow[t]{2}{*}{ Element } & \multicolumn{2}{|c|}{ CS 31080-095 } & \multicolumn{2}{|c|}{ CS 22958-042 } & \multicolumn{2}{|c|}{ CS 29528-041 } & \multirow{2}{*}{$\begin{array}{r}\text { Sun } \\
\log (\epsilon)\end{array}$} \\
\hline & $\log (\epsilon)$ & {$[\mathrm{X} / \mathrm{Fe}]$} & $\log (\epsilon)$ & {$[\mathrm{X} / \mathrm{Fe}]$} & $\log (\epsilon)$ & {$[\mathrm{X} / \mathrm{Fe}]$} & \\
\hline$\overline{\mathrm{Li}}$ & $1.73 \pm 0.05$ & & $<0.6$ & & $1.71 \pm 0.05$ & & 1.10 \\
\hline $\mathrm{C}(\mathrm{CH})$ & $8.25 \pm 0.01$ & $+2.69 \pm 0.14$ & $8.70 \pm 0.01$ & $+3.15 \pm 0.12$ & $6.70 \pm 0.08$ & $+1.59 \pm 0.15$ & 8.41 \\
\hline $\mathrm{C}(\mathrm{C} 2)$ & $8.35 \pm 0.01$ & $+2.79 \pm 0.14$ & $8.70 \pm 0.01$ & $+3.15 \pm 0.12$ & $\ldots$ & & 8.41 \\
\hline $\mathrm{N}(\mathrm{NH})$ & & & $7.05 \pm 0.02$ & $+2.10 \pm 0.12$ & $7.50 \pm 0.1$ & $+3.00 \pm 0.15$ & 7.80 \\
\hline $\mathrm{N}(\mathrm{CN})$ & $5.65 \pm 0.01$ & $+0.70 \pm 0.11$ & $7.10 \pm 0.01$ & $+2.15 \pm 0.11$ & $7.57 \pm 0.06$ & $+3.07 \pm 0.13$ & 7.80 \\
\hline $\mathrm{O}(\mathrm{LTE})$ & $8.17 \pm 0.05$ & $+2.35 \pm 0.12$ & $7.17 \pm 0.05$ & $+1.35 \pm 0.11$ & $<6.77$ & $<+1.40 \pm 0.10$ & 8.67 \\
\hline O(NLTE) & $8.05 \pm 0.05$ & $+2.23 \pm 0.12$ & $6.94 \pm 0.05$ & $+1.12 \pm 0.11$ & $<6.77$ & $<+1.40 \pm 0.10$ & 8.67 \\
\hline $\mathrm{Na}(\mathrm{LTE})$ & $3.20 \pm 0.05$ & $-0.28 \pm 0.12$ & $6.30 \pm 0.10$ & $+2.82 \pm 0.15$ & $4.23 \pm 0.05$ & $+1.20 \pm 0.11$ & 6.33 \\
\hline $\mathrm{Na}(\mathrm{NLTE})$ & $3.00 \pm 0.05$ & $-0.48 \pm 0.12$ & $6.10 \pm 0.10$ & $+2.62 \pm 0.15$ & $4.02 \pm 0.05$ & $+1.00 \pm 0.11$ & 6.33 \\
\hline Mg I & 5.38 & $+0.65 \pm 0.12$ & $5.05 \pm 0.04$ & $+0.32 \pm 0.15$ & $4.68 \pm 0.01$ & $+0.40 \pm 0.11$ & 7.58 \\
\hline $\mathrm{Al}$ (LTE) & 2.67 & $-0.95 \pm 0.11$ & 2.77 & $-0.85 \pm 0.10$ & 2.77 & $-0.85 \pm 0.10$ & 6.47 \\
\hline $\mathrm{Al}$ (NLTE) & 3.32 & $-0.30 \pm 0.11$ & 3.32 & $-0.20 \pm 0.10$ & 3.32 & $-0.20 \pm 0.10$ & 6.47 \\
\hline Si I & 4.75 & $+0.05 \pm 0.15$ & 4.85 & $+0.15 \pm 0.10$ & 4.05 & $-0.20 \pm 0.10$ & 7.55 \\
\hline $\mathrm{Ca} \mathrm{I}$ & $3.68 \pm 0.05$ & $+0.17 \pm 0.13$ & $3.87 \pm 0.06$ & $+0.36 \pm 0.15$ & $3.46 \pm 0.03$ & $+0.40 \pm 0.15$ & 6.36 \\
\hline Sc II & $0.30 \pm 0.05$ & $-0.02 \pm 0.14$ & $0.37 \pm 0.05$ & $+0.05 \pm 0.11$ & $0.13 \pm 0.03$ & $+0.26 \pm 0.15$ & 3.17 \\
\hline Ti I & $2.59 \pm 0.03$ & $+0.42 \pm 0.16$ & 2.60 & $+0.43 \pm 0.10$ & $2.32 \pm 0.04$ & $+0.40 \pm 0.15$ & 5.02 \\
\hline Ti II & $2.39 \pm 0.01$ & $+0.22 \pm 0.11$ & $2.36 \pm 0.07$ & $+0.19 \pm 0.14$ & $2.12 \pm 0.007$ & $+0.40 \pm 0.10$ & 5.02 \\
\hline V I & $1.15 \pm 0.05$ & $+0.00 \pm 0.12$ & 1.10 & $-0.05 \pm 0.10$ & 0.70 & $+0.00 \pm 0.15$ & 4.00 \\
\hline $\mathrm{Cr} \mathrm{I}$ & $2.84 \pm 0.03$ & $+0.02 \pm 0.12$ & $2.67 \pm 0.04$ & $-0.15 \pm 0.11$ & $2.20 \pm 0.01$ & $-0.17 \pm 0.11$ & 5.67 \\
\hline Mn I & $2.33 \pm 0.05$ & $-0.21 \pm 0.13$ & $2.33 \pm 0.05$ & $-0.21 \pm 0.14$ & $1.59 \pm 0.07$ & $-0.50 \pm 0.15$ & 5.39 \\
\hline $\mathrm{Fe} \mathrm{I}$ & $4.65 \pm 0.003$ & $-0.00 \pm 0.10$ & $4.65 \pm 0.04$ & $+0.00 \pm 0.10$ & $4.20 \pm 0.002$ & $+0.00 \pm 0.10$ & 7.50 \\
\hline $\mathrm{Fe}$ II & $4.66 \pm 0.02$ & $-0.05 \pm 0.11$ & $4.57 \pm 0.09$ & $-0.08 \pm 0.12$ & $4.20 \pm 0.02$ & $+0.00 \pm 0.10$ & 7.50 \\
\hline Co I & $2.38 \pm 0.03$ & $+0.31 \pm 0.11$ & $2.27 \pm 0.14$ & $+0.20 \pm 0.18$ & $1.62 \pm 0.04$ & $+0.00 \pm 0.15$ & 4.92 \\
\hline $\mathrm{Ni} I$ & $3.49 \pm 0.03$ & $+0.09 \pm 0.16$ & $3.31 \pm 0.10$ & $-0.09 \pm 0.16$ & $2.95 \pm 0.01$ & $+0.00 \pm 0.12$ & 6.25 \\
\hline Zn I & $2.33 \pm 0.08$ & $+0.58 \pm 0.15$ & $\ldots$ & $\ldots$ & $\ldots$ & $\ldots$ & 4.60 \\
\hline Sr II & $-0.29 \pm 0.05$ & $-0.41 \pm 0.12$ & -0.53 & $-0.20 \pm 0.11$ & -0.53 & $-0.20 \pm 0.10$ & 2.97 \\
\hline Y II & $-0.96 \pm 0.05$ & $-0.35 \pm 0.13$ & $\ldots$ & & $\ldots$ & $\ldots$ & 2.24 \\
\hline Ba II & $0.05 \pm 0.05$ & $+0.77 \pm 0.15$ & $<-1.40$ & $<-0.53 \pm 0.16$ & -0.23 & $+0.97 \pm 0.10$ & 2.13 \\
\hline${ }^{12} \mathrm{C} /{ }^{13} \mathrm{C}$ & $>40$ & & $9.0 \pm 2.0$ & $\ldots$ & $\cdots$ & $\ldots$ & $\ldots$ \\
\hline
\end{tabular}

values of Ba enrichment fall below the level suggested by Beers \& Christlieb for the CEMP-s classification ([Ba/Fe $>+1.0)$, but they do satisfy the Ryan et al. (2005) criterion for Ba-rich stars, $[\mathrm{Ba} / \mathrm{Fe}]>+0.50$, which is also adopted by Aoki et al. (2006a). Note that the $\mathrm{Sr}$ abundances in these two stars are quite low, $[\mathrm{Sr} / \mathrm{Fe}]=-0.41(\mathrm{CS} 31080-095)$ and $[\mathrm{Sr} / \mathrm{Fe}]=-0.20$ (CS 29528-041).

CS 31080-095 and CS 22958-042 are more C rich than $\mathrm{N}$ rich. In contrast, CS 29528-041 is one of the most $\mathrm{N}$-enhanced stars yet found among CEMP stars $([\mathrm{N} / \mathrm{Fe}]=+3.0)$. In the following subsections we consider the nature of the light elements, $\mathrm{O}$ and other $\alpha$ elements, the odd-Z elements, the ironpeak elements, and the neutron-capture elements in our three program stars.

\subsection{Li, $C, N$, and the ${ }^{12} C /{ }^{13} \mathrm{C}$ ratio}

We detect the Li I $670.7 \mathrm{~nm}$ doublet in both CS 31080-095 and CS 29528-041 (see Fig. 1); $A(\mathrm{Li}) \sim 1.7$ is derived for both of these stars. The Li line is not detected in CS 22958-042; we derive an upper limit of $A(\mathrm{Li})<0.6(3 \sigma)$. It would clearly be of interest to obtain higher- $\mathrm{S} / \mathrm{N}$ spectra of this star in order to strengthen this limit.

Both CS 31080-095 and CS 22958-042 exhibit very large $\mathrm{C}$ enhancements; $[\mathrm{C} / \mathrm{Fe}]=+2.75$ and $[\mathrm{C} / \mathrm{Fe}]=+3.15$, respectively, as estimated from the average of results derived from the $\mathrm{CH}$ and $\mathrm{C}_{2}$ features. In CS 29528-041 the $\mathrm{C}_{2}$ lines are not detected; only the weak $\mathrm{CH}$ lines could be used to estimate $[\mathrm{C} / \mathrm{Fe}]$ in this star.

Nitrogen abundances for our stars are obtained from the CN $388.3 \mathrm{~nm}$ band (see Fig. 2); for CS 22958-042 and

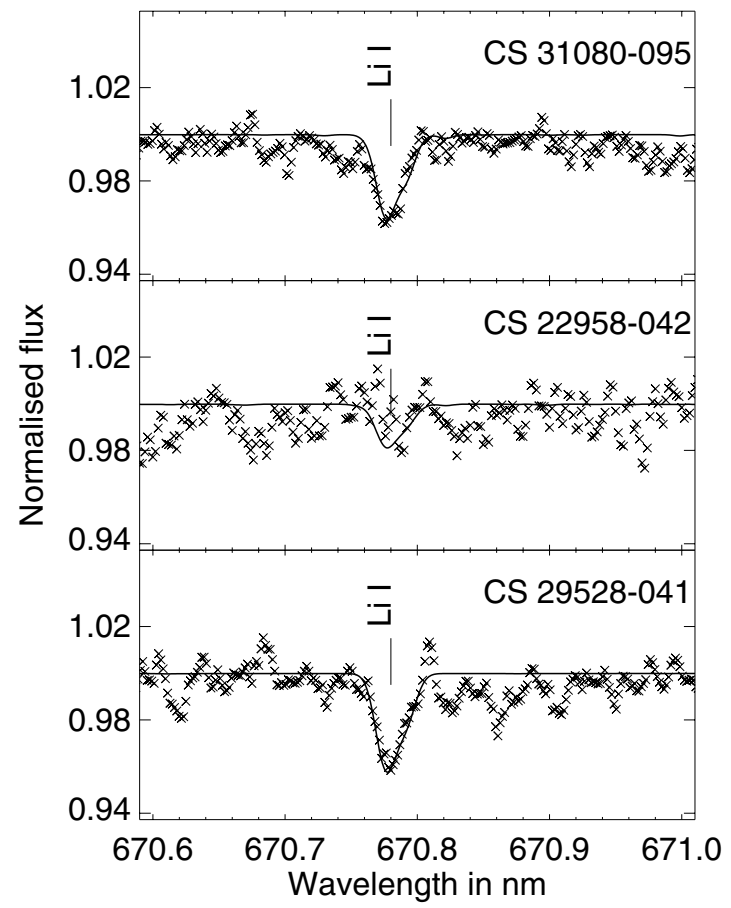

Fig. 1. Observed (crosses) and synthetic spectra (lines) of CS 31080-095, CS 22958-042, and CS 29528-041 in the region of the $\mathrm{Li}$ I $670.7 \mathrm{~nm}$ doublet. $\mathrm{Li}$ is not detected in CS 22958-042.

CS 29528-041 we also use the NH band at $336 \mathrm{~nm}$. The NH and $\mathrm{CN}$ lines yield slightly different abundances; we adopt the results from the $\mathrm{CN}$ lines, which have better $\mathrm{S} / \mathrm{N}$. 


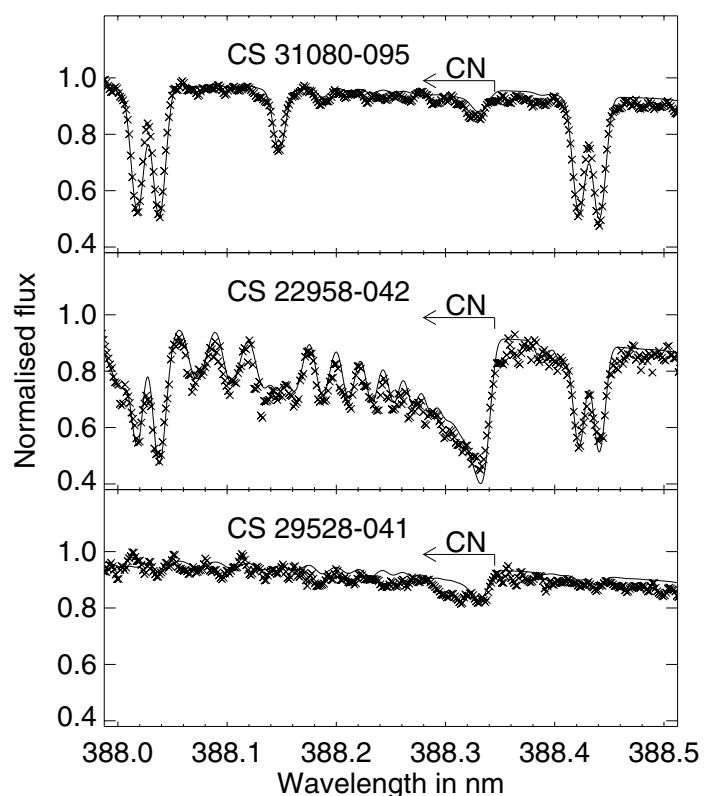

Fig. 2. Observed and synthetic spectra of our stars around the $388.3 \mathrm{~nm}$ $\mathrm{CN}$ band (symbols as in Fig. 1). The strong CN bands in CS 22958-042 indicate a larger $\mathrm{N}$ excess than in CS 31080-095. In CS 29528-041 the $\mathrm{CN}$ bands are weak, but the derived $[\mathrm{N} / \mathrm{H}]$ is high because $[\mathrm{C} / \mathrm{H}]$ is low and $T_{\text {eff }}$ is high. The $336 \mathrm{~nm} \mathrm{NH}$ bands are also detected in CS 29528-041 and CS 22958-042 (not shown).

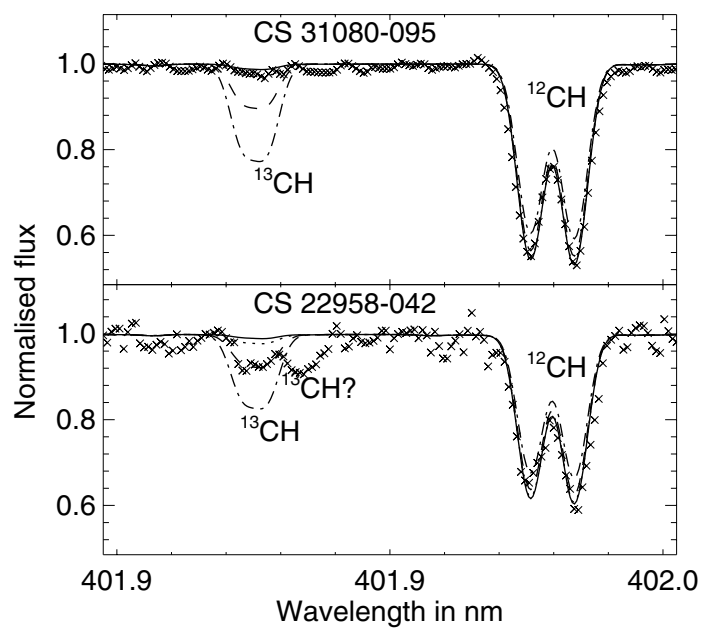

Fig. 3. Observed spectra (crosses) for the two stars with meaningful measurements or limits on the ${ }^{12} \mathrm{C} /{ }^{13} \mathrm{C}$ ratio. Solid, dotted, dashed, and dash-dotted lines show our synthetic spectra for ${ }^{12} \mathrm{C} /{ }^{13} \mathrm{C}=99,40,9$, and 3, respectively. For CS $29528-041$ all ${ }^{12} \mathrm{C} /{ }^{13} \mathrm{C}$ ratios between 3 and 99 are within a $2 \sigma$ detection limit; hence, no ${ }^{12} \mathrm{C} /{ }^{13} \mathrm{C}$ is given for this star.

The ${ }^{13} \mathrm{CH}$ lines at $401.9 \mathrm{~nm}$ (see Fig. 3) and the ${ }^{13} \mathrm{C}$, ${ }^{12} \mathrm{C} 474.0 \mathrm{~nm}$ lines are used to derive ${ }^{12} \mathrm{C} /{ }^{13} \mathrm{C}$ ratios. Only a weak lower limit on the ${ }^{12} \mathrm{C} /{ }^{13} \mathrm{C}$ ratio resulted for CS 31080-095, while a clear determination of ${ }^{12} \mathrm{C} /{ }^{13} \mathrm{C}=9.0$ is found for CS 22958-042. The high $T_{\text {eff }}$ and moderate $\mathrm{C}$ enhancement in CS 29528-041 make the ${ }^{13} \mathrm{C}$ features very weak, and prevent us from placing any useful limits on ${ }^{12} \mathrm{C} /{ }^{13} \mathrm{C}$ in this star.

\section{2. $O$ and other $\alpha$ elements}

We estimate $\mathrm{O}$ abundances by LTE analysis of the O I $777.4 \mathrm{~nm}$ triplet, recognising that it is affected by both NLTE
(Kiselman 1991; Takeda 1994; Gratton et al. 1999; Kiselman 2001) and 3D effects (Asplund 2005). In CS 31080-095 the triplet lines are quite strong; we derive $[\mathrm{O} / \mathrm{Fe}]=+2.35$. We do not detect the O I lines in CS 29528-041; hence we only obtain an upper limit of $[\mathrm{O} / \mathrm{Fe}]<+1.4(3 \sigma)$. The $[\mathrm{O} / \mathrm{Fe}]$ values in Table 3 include the NLTE corrections by Gratton et al. (1999).

We find $[\mathrm{Mg} / \mathrm{Fe}] \sim+0.65$ in CS $31080-095$ and $[\mathrm{Mg} / \mathrm{Fe}]=$ +0.32 and +0.4 in CS 22958-042 and CS 29528-041, respectively, similar to most other VMP stars. Si is overabundant by +0.05 to +0.15 dex in CS 31080-095 and CS 22958-042, and $\mathrm{Ca}$ is overabundant in all three stars by +0.2 to +0.4 dex.

\subsection{The odd-Z elements}

Sodium abundances for our stars are determined using the $\mathrm{Na}$ I D lines, which can be affected by NLTE. We have applied the NLTE corrections by Baumüller et al. (1998), as listed in their Table 2. The Na I D lines in CS 22958-042 are well separated from the significant interstellar component, due to the high radial velocity of this star. In CS 29528-041 the Na I D1 line merges with the interstellar Na I D2 line, so we derive the $\mathrm{Na}$ abundance from the D2 line alone. Aluminium is underabundant in all three stars after an NLTE correction of +0.65 dex (Baumüller \& Gehren 1997).

\subsection{The iron-peak elements}

The abundances of $\mathrm{Ti}, \mathrm{Sc}, \mathrm{V}, \mathrm{Cr}, \mathrm{Mn}, \mathrm{Co}$, and $\mathrm{Ni}$ are similar in all three stars, and close to the values reported for halo stars in the same metallicity range (e.g., Cayrel et al. 2004).

\subsection{The neutron-capture elements}

We derive strontium abundance estimates for our stars from the $\mathrm{Sr}$ II $407.7 \mathrm{~nm}$ and $421.5 \mathrm{~nm}$ lines. All three of our program stars exhibit $\mathrm{Sr}$ underabundances, from -0.2 to -0.4 dex. A weak Y II line at $377.4 \mathrm{~nm}$ is also detected in CS 31080-095, from which we derive $[\mathrm{Y} / \mathrm{Fe}]=-0.35$. Barium is moderately overabundant in both CS 31080-095 and CS 29528-041, while it appears to be quite low in CS 22958-042. Barium abundances were estimated from the Ba II $455.4 \mathrm{~nm}$ and $493.4 \mathrm{~nm}$ lines. For CS 22958-042, we could not detect any Ba II lines at all, and derive an upper limit of $[\mathrm{Ba} / \mathrm{Fe}]<-0.53(3 \sigma)$, adopting the hyperfine splitting by McWilliam (1998) for Ba II and assuming a solar isotopic composition.

\section{Discussion}

Here we discuss the results for our three program stars in the context of the CEMP class as a whole. Table 4 lists elemental abundances and ${ }^{12} \mathrm{C} /{ }^{13} \mathrm{C}$ ratios for CEMP stars from the recent literature, grouped into the CEMP-no, CEMP-s, and CEMP-r/s classes. Our two moderately Ba-enhanced stars form a fourth group, which we call CEMP-no/s stars.

We first discuss the $\mathrm{C}$ and $\mathrm{N}$ abundances for our CEMP stars. We next describe the nature of the oxygen abundance in these stars, followed by a discussion of $\mathrm{Na}, \mathrm{Mg}$, and other heavier elements. The variation of the ${ }^{12} \mathrm{C} /{ }^{13} \mathrm{C}$ ratio with $\mathrm{Li}$ abundance is then considered. 
Table 4. Elemental abundances and ${ }^{12} \mathrm{C} /{ }^{13} \mathrm{C}$ ratios for CEMP stars.

\begin{tabular}{|c|c|c|c|c|c|c|c|c|c|c|c|c|}
\hline Star & $T_{\text {eff }}$ & $\log g$ & {$[\mathrm{Fe} / \mathrm{H}]$} & [C/Fe] & {$[\mathrm{O} / \mathrm{Fe}]$} & [N/Fe] & {$[\mathrm{Mg} / \mathrm{Fe}]$} & {$[\mathrm{Sr} / \mathrm{Fe}]$} & {$[\mathrm{Ba} / \mathrm{Fe}]$} & ${ }^{12} \mathrm{C} /{ }^{13} \mathrm{C}$ & $A(\mathrm{Li})$ & Ref \\
\hline \multicolumn{13}{|c|}{ CEMP-no stars ${ }^{a}$} \\
\hline CS 22877-001 & 5100 & 2.2 & -2.71 & +1.0 & $\ldots$ & +0.0 & +0.29 & -0.12 & -0.49 & $>10$ & $\ldots$ & 4 \\
\hline CS 22949-037 & 4900 & 1.7 & -3.79 & +1.17 & +1.98 & +2.57 & +1.56 & +0.33 & -0.58 & 4 & $\ldots$ & 10,17 \\
\hline CS 22957-027 & 5100 & 1.4 & -3.11 & +2.37 & $\ldots$ & +1.62 & +0.69 & -0.56 & -1.23 & 8 & $\ldots$ & 3,19 \\
\hline CS 22958-042 & 6250 & 3.5 & -2.85 & +3.15 & +1.12 & +2.15 & +0.32 & -0.2 & $<-0.53$ & 9 & $<0.6$ & TP \\
\hline CS 29498-043 & 4600 & 1.2 & -3.53 & +2.09 & +2.43 & +2.27 & +1.75 & -0.47 & -0.46 & 6 & $\cdots$ & 3,7 \\
\hline CS 29502-092 & 5000 & 2.1 & -2.77 & +1.0 & $\cdots$ & +1.0 & +0.37 & -0.40 & -0.82 & 20 & $\cdots$ & 4 \\
\hline G 77-61 & 4000 & 5.0 & -4.0 & +3.2 & +2.2 & +2.2 & +0.49 & $\ldots$ & $\ldots$ & 5 & $<1.0$ & 18 \\
\hline HE 0007-1832 & 6515 & 3.8 & -2.65 & +2.55 & $\cdots$ & +1.85 & $\ldots$ & $\cdots$ & +0.16 & 10 & $\cdots$ & 9 \\
\hline HE 0107-5240 & 5180 & 2.2 & -5.3 & +4.1 & $\ldots$ & +2.3 & $\ldots$ & $\ldots$ & $\ldots$ & $\ldots$ & $\ldots$ & 8 \\
\hline HE 1327-2326 & 6180 & 3.7 & -5.6 & +4.1 & 2.8 & +4.5 & $\cdots$ & +1.1 & $<+1.5$ & $\cdots$ & $<1.5$ & $11,11 \mathrm{a}, 21$ \\
\hline \multicolumn{13}{|c|}{ CEMP-no/s stars ${ }^{b}$} \\
\hline CS 29528-041 & 6166 & 4.0 & -3.30 & +1.59 & $<1.40$ & +3.07 & +0.4 & -0.2 & +0.97 & $\ldots$ & 1.71 & $\mathrm{TP}$ \\
\hline CS 31080-095 & 6100 & 4.5 & -2.85 & +2.69 & 2.23 & +0.70 & +0.65 & -0.41 & +0.77 & $>40$ & 1.73 & $\mathrm{TP}$ \\
\hline \multicolumn{13}{|l|}{ CEMP-s stars ${ }^{c}$} \\
\hline CS 22880-074 & 5850 & 3.8 & -1.93 & +1.3 & $\cdots$ & -0.1 & $\cdots$ & +0.39 & +1.31 & $>40$ & $\cdots$ & 3,19 \\
\hline CS 22942-019 & 5000 & 2.4 & -2.64 & +2.0 & $\cdots$ & +0.3 & $\ldots$ & +1.7 & +1.92 & 8 & $\cdots$ & 3,19 \\
\hline CS 30301-015 & 4750 & 0.8 & -2.64 & +1.6 & $\cdots$ & +0.6 & $\cdots$ & +0.3 & +1.45 & 6 & $\cdots$ & 3 \\
\hline \multicolumn{13}{|c|}{ CEMP-r/s stars ${ }^{d}$} \\
\hline CS 22898-027 & 6250 & 3.7 & -2.26 & +2.2 & $\cdots$ & +0.9 & $\cdots$ & +0.92 & +2.23 & $>20$ & 2.10 & 3,19 \\
\hline CS 22948-027 & 4600 & 1.0 & -2.57 & +2.43 & $\ldots$ & +1.75 & +0.31 & +0.90 & +2.26 & 10 & 0.30 & 3,19 \\
\hline \multirow[t]{2}{*}{ CS 29497-030 } & 6650 & 3.5 & -2.70 & +2.38 & +1.67 & +1.88 & +0.64 & +0.84 & +2.17 & $>10$ & $<1.10$ & 20 \\
\hline & 7000 & 4.1 & -2.57 & +2.30 & +1.48 & +2.12 & +0.44 & +1.34 & +2.32 & $\ldots$ & $\ldots$ & 13 \\
\hline CS 29497-034 & 4800 & 1.8 & -2.90 & +2.63 & $\ldots$ & +2.38 & +0.72 & +1.00 & +2.03 & 12 & 0.10 & 12 \\
\hline CS 29526-110 & 6500 & 3.2 & -2.38 & +2.2 & $\ldots$ & +1.4 & $\ldots$ & +0.88 & +2.11 & $\ldots$ & $\ldots$ & 5 \\
\hline CS 31062-012 & 5600 & 3.0 & -2.55 & +2.1 & $\cdots$ & +1.2 & $\ldots$ & +0.30 & +1.98 & 8 & $\ldots$ & 3,6 \\
\hline CS 31062-050 & 5600 & 3.0 & -2.33 & +2.0 & $\ldots$ & +1.2 & +0.84 & +0.91 & +2.80 & 8 & $\cdots$ & 3,6 \\
\hline HE 0024-2523 & 6625 & 4.3 & -2.67 & +2.6 & +0.40 & +2.1 & +0.73 & +0.34 & +1.46 & 6 & 1.50 & 15 \\
\hline LP 625-44 & 5500 & 2.8 & -2.71 & +2.1 & $\ldots$ & +1.0 & $\ldots$ & +1.15 & +2.74 & 20 & 0.40 & 1,2 \\
\hline LP 706-7 & 6250 & 4.5 & -2.74 & +2.15 & $\cdots$ & +1.80 & $\cdots$ & +0.15 & +2.01 & $>15$ & 2.09 & 3,16 \\
\hline HE 0143-0441 & 6370 & 4.4 & -2.16 & +1.66 & $\ldots$ & -0.04 & $\ldots$ & $\ldots$ & +2.31 & 10 & $\cdots$ & 9 \\
\hline HE 0338-3945 & 6160 & 4.1 & -2.42 & +2.13 & +1.40 & +1.55 & +0.30 & +0.74 & +2.41 & 10 & $\ldots$ & 14 \\
\hline HE 2148-1247 & 6380 & 4.3 & -2.3 & +1.91 & $\ldots$ & +1.65 & $\ldots$ & $\ldots$ & +2.36 & 10 & $\ldots$ & 9 \\
\hline
\end{tabular}

${ }^{a}[\mathrm{C} / \mathrm{Fe}]>+1.0$ and $[\mathrm{Ba} / \mathrm{Fe}]<0.0$ (Beers \& Christlieb 2005); ${ }^{b}[\mathrm{C} / \mathrm{Fe}]>+1.0$ and $+0.5<[\mathrm{Ba} / \mathrm{Fe}]<+1.0 ;{ }^{c}[\mathrm{C} / \mathrm{Fe}]>+1.0,[\mathrm{Ba} / \mathrm{Fe}]>+1.0$, and $[\mathrm{Ba} / \mathrm{Eu}]>+0.5$ (Beers \& Christlieb 2005); ${ }^{d}[\mathrm{C} / \mathrm{Fe}]>+1.0$ and $0.0<[\mathrm{Ba} / \mathrm{Fe}]<+0.5$ (Beers \& Christlieb 2005).

Ref.: (1) Aoki et al. (2000); (2) Aoki et al. (2001); (3) Aoki et al. (2002a); (4) Aoki et al. (2002b); (5) Aoki et al. (2002c); (6) Aoki et al. (2003); (7) Aoki et al. (2004); (8) Christlieb et al. (2002); (9) Cohen et al. (2003); (10) Depagne et al. (2002); (11) Frebel et al. (2005); (11a)Frebel et al. (2006); (12) Hill et al. (2000); (13) Ivans et al. (2005); (14) Jonsell et al. (2006); (15) Lucatello et al. (2003); (16) Norris et al. (1997); (17) Norris et al. (2002); (18) Plez et al. (2005); (19) Preston \& Sneden (2001); (20) Sivarani et al. (2004); (21) Aoki et al. (2006b); (TP) This paper.

\section{1. $C$ and $N$ abundances in CEMP stars}

CS 31080-095 and CS 22958-042 exhibit strong C and N enhancements, similar to most other CEMP stars. CS 22958-042 stands out as one of the most carbon-rich of the known CEMP stars, while CS 29528-041 exhibits one of the largest $\mathrm{N}$ abundances yet observed in CEMP stars.

Figure 4 shows a broad overall correlation of the $\mathrm{C}$ and $\mathrm{N}$ overabundances in most CEMP stars. Note that the "carbonnormal" stars from Spite et al. (2005), shown in the lower left part of the figure, display a clear anti-correlation between $\mathrm{C}$ and $\mathrm{N}$ due to mixing with $\mathrm{CNO}$-cycled material (see also Spite et al. 2006).

$\mathrm{C}$ and $\mathrm{N}$ have distinct nucleosynthetic formation paths: $\mathrm{C}$ is made by He burning in the triple- $\alpha$ process, while $\mathrm{N}$ production requires $\mathrm{H}$ burning, i.e., two proton captures on ${ }^{12} \mathrm{C}$. Because the production site of primary $\mathrm{C}$, the He-burning zone, is void of protons, primary $\mathrm{C}$ must be mixed out of the He-burning region and into the H-rich outer layers under conditions that are still hot enough for effective proton capture. Thus, in a sense, it is not surprising to see high $\mathrm{C}$ and $\mathrm{N}$ together.
However, in stellar models it is very difficult to realize the specific conditions for $\mathrm{He}$ and $\mathrm{H}$ burning and mixing that can produce the observed abundance patterns quantitatively. HotBottom Burning (HBB) in intermediate-mass stars has long been the only solid candidate site for primary $\mathrm{N}$ production. Carbon is produced in the He-shell flashes and dredged up into the envelope after the thermal pulse. During the interpulse phase between He-shell flashes the convective envelope connects with the outer layers of the $\mathrm{H}$-burning shell, and the $\mathrm{C}$ in the envelope is transformed into $\mathrm{N}$.

As shown, for example, by the 4-6 $M_{\odot}$ models of Herwig (2004), the mass-averaged ejecta of such of VMP intermediatemass stars can reach $[\mathrm{N} / \mathrm{Fe}] \sim+2$ to +3 . Hot-bottom burning in intermediate-mass stars is characterized by very efficient conversion of $\mathrm{C}$ and $\mathrm{O}$ into $\mathrm{N}$. This finding is model independent. The $\mathrm{C}$ overabundance in 4-6 $M_{\odot}$ VMP models is in the range $[\mathrm{C} / \mathrm{Fe}] \sim+1$ to +2 .

For stars that show no sign of the main component of the s-process or of being binaries, one must consider other possible sources for the observed $\mathrm{C}$ and $\mathrm{N}$ overabundances. Massive stars have been often dismissed in the past because of the 


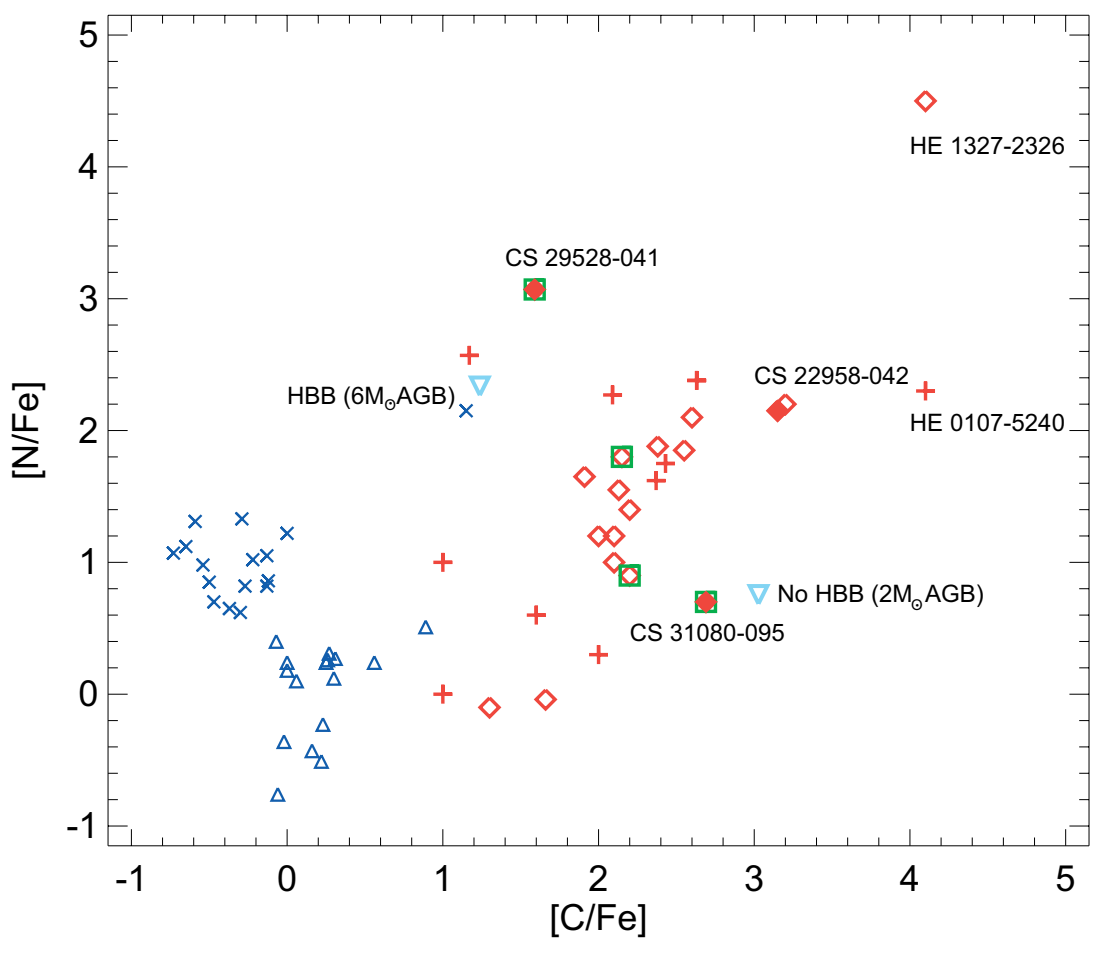

Fig. 4. $[\mathrm{N} / \mathrm{Fe}]$ vs. $[\mathrm{C} / \mathrm{Fe}]$ for CEMP stars from the recent literature (Table 4). The $\times$ and $\triangle$ symbols represent the mixed and unmixed giants from Spite et al. (2005); the + and $\diamond$ signs represent stars with $\log g<$ 3.5 and $\log g>3.5$, respectively. Stars with significant Li detections are surrounded by a $\square$ (filled symbols: this paper). The AGB model predictions by Herwig (2004) for $[\mathrm{Fe} / \mathrm{H}]=-2.3$ and initial masses $6 M_{\odot}(\mathrm{HBB})$ and $2 M_{\odot}$ (non-HBB) are shown by $\nabla$ symbols. HE $0107-$ 5240 and HE 1327-2326 are the two known HMP stars. inability of standard models to produce primary N. This inability is related to the above-mentioned necessity of mixing Heburning products $(\mathrm{C})$ out into the $\mathrm{H}$-burning region.

However, the recent models by Hirschi et al. (2006) and Meynet et al. (2006) show that massive, MMP $([\mathrm{Fe} / \mathrm{H}]<-6.0)$ stars that include the effect of rotationally-induced mixing have the desired ability to produce large amounts of primary N (which is required to match the observed behavior of [N/O] at low metallicity, even among "carbon normal" stars; see Chiappini et al. 2006). The $\mathrm{C}$ and $\mathrm{N}$ abundance signatures of rotating $60 M_{\odot}$ models and the E-AGB envelopes of intermediate-mass stars (7 $M_{\odot}$ ) are quite similar (Meynet et al. 2006).

$\mathrm{C}$ and $\mathrm{N}$ abundances from HBB models with initial mass $2 M_{\odot}$ and non-HBB AGB models with initial mass $6 M_{\odot}$ (Herwig 2004) are indicated in Fig. 4. Note that because these (as well as the massive-star model predictions) are primary yields, they shift along diagonals through zero in the $[\mathrm{C} / \mathrm{Fe}]-[\mathrm{N} / \mathrm{Fe}]$ diagram as a function of $[\mathrm{Fe} / \mathrm{H}]$. There is more than a one dex difference in $[\mathrm{Fe} / \mathrm{H}]$ between the HBB models and the non-HBB models. These two classes of models seem to delimit the region occupied by the CEMP stars.

Most CEMP stars in Fig. 4 are located in an intermediate regime, showing larger $\mathrm{N}$ and smaller $\mathrm{C}$ overabundances than predicted by the the 2 and $3 M_{\odot}$ AGB models, but less $\mathrm{N}$ and more $\mathrm{C}$ than predicted by the HBB AGB models. One of our program stars, CS 22958-042, falls into this category as well. Note, however, that CS 31080-095 exhibits [N/Fe] and [C/Fe] ratios in almost perfect agreement with the non-HBB models.

Most other CEMP stars exhibit too strong $\mathrm{N}$ to be explained by standard low-mass AGB companions. It is interesting to note that the extra mixing, or cool-bottom burning, on the AGB that has been mentioned above in the context of $\mathrm{Li}$ abundances, could also increase the $\mathrm{N}$ abundance in 2 and $3 M_{\odot}$ VMP AGB stars, possibly to the extent required by observations. Unfortunately, no quantitative models are currently available that consider this effect.

CS 29528-041 is one of the very few CEMP stars that exhibits the high $\mathrm{N}$ and low $\mathrm{C}$ expected from HBB AGB stars.
Johnson et al. $(2005,2006)$ have searched for stars with low $[\mathrm{C} / \mathrm{N}]$ ratios as predicted by current AGB models, using near$\mathrm{UV} \mathrm{NH}$ lines rather than the $\mathrm{CN}$ lines which may be weak in low- $[\mathrm{C} / \mathrm{N}]$ stars; no obvious low- $[\mathrm{C} / \mathrm{N}]$ candidates were found.

This apparent paucity of N-rich EMP stars is a matter of concern. If the hypothesis that CEMP-s stars are mass-transfer binaries with AGB stars as donors is correct, then why do we not see the occasional Nitrogen-Enhanced Metal-Poor (NEMP) star that had a HBB AGB star as a companion? The $\mathrm{C}$ and $\mathrm{N}$ abundances suggest that CS 29528-041 is at least one candidate for these "missing" NEMP stars.

\subsection{Oxygen}

The C and $\mathrm{N}$ overabundances of CS 31080-095 and CS 22958-042 are typical of CEMP stars in general; however, they also exhibit significant $\mathrm{O}$ overabundances $([\mathrm{O} / \mathrm{Fe}]=+2.35$ and +1.35 , respectively). Near solar metallicity, the production of $\mathrm{O}$ in AGB stars is negligible. It is therefore at first sight surprising to see large overabundances of $\mathrm{O}$ in stars that are considered to be substantially polluted by AGB stars.

However, for $[\mathrm{Fe} / \mathrm{H}] \leq-2$, AGB models produce primary $\mathrm{O}$ quite efficiently. In the model yields by Herwig (2004), the $\mathrm{O}$ overabundances range from $[\mathrm{O} / \mathrm{Fe}]=+1.84$ for the $2 M_{\odot}$ case to $[\mathrm{O} / \mathrm{Fe}]=+0.39$ for the $6 M_{\odot}$ case. Again, because these are primary yields, they would to first order increase at lower $[\mathrm{Fe} / \mathrm{H}]$. Note that the $\mathrm{O}$ overabundance in the yields of Herwig (2004) should be a minimum, as they do not take convective overshoot into account; this would increase the $\mathrm{O}$ abundance in the AGB intershell region and increase the yields even further.

The $\mathrm{O}$ abundance in the He-shell flash convection zone is about $1-2 \%$ by mass, almost independent of metallicity. At solar metallicity this is about the initial abundance level. Thus, any primary $\mathrm{O}$ produced in the $\mathrm{He}$-shell flash convection zone that is dredged up into the envelope will not significantly increase the surface $\mathrm{O}$ abundance. However, at extremely low metallicity, the envelope initially contains much less $\mathrm{O}$ than the primary 
$\mathrm{O}$ contained in the He-flash intershell material that is dredged up into the envelope. Therefore, even a seemingly small $\mathrm{O}$ abundance of $1-2 \%$ in the intershell region leads to a significant $\mathrm{O}$ excess compared to the initial $\mathrm{O}$ abundance. The existence of such an enhanced intershell $\mathrm{O}$ abundance is supported by observations of hot H-deficient central stars of planetary nebulae (Werner \& Herwig 2006).

For CS 29528-041 the low upper limit for O is consistent with HBB AGB predictions. For CS 31080-095 and CS 22958-042 the O abundance fits into the picture where the observed abundances are produced by non-HBB AGB stars.

\subsection{Sodium}

Sodium is extremely important for understanding the nuclear production site of CEMP-star abundances. A small amount of secondary $\mathrm{Na}$ can be made by $\mathrm{C}$-burning in massive stars. However, with the large overabundances of $\mathrm{Na}$ observed in CS 22958-042 $([\mathrm{Na} / \mathrm{Fe}]=+2.82)$ and CS 29528-041 $([\mathrm{Na} / \mathrm{Fe}]=+1.20)$ and many other CEMP stars, we need to look for a primary source of $\mathrm{Na}$.

As explained above, primary production of ${ }^{14} \mathrm{~N}$ is difficult because it requires the He-burning ashes to be exposed to -burning again, which requires mixing. For $\mathrm{Na}$ this concept must be taken two steps further. Once there is primary ${ }^{14} \mathrm{~N}$ from successive $\mathrm{He}$ and $\mathrm{H}$ burning, the ${ }^{14} \mathrm{~N}$ must be brought back into the region of He burning in order to capture two $\alpha$-particles. The result is primary ${ }^{22} \mathrm{Ne}$, which must then capture either a neutron or a proton in order to make ${ }^{23} \mathrm{Na}$. Through the recurrent, interconnected He-shell flash and dredge-up events, AGB stars provide a natural environment for producing significant amounts of primary $\mathrm{Na}$. In fact, AGB stars of all masses, whether HBB or not, are predicted to produce primary $\mathrm{Na}$.

Primary ${ }^{14} \mathrm{~N}$ exists in the ashes of the H-burning shell after a few thermal pulses with dredge-up. This ${ }^{14} \mathrm{~N}$ is engulfed by the next He-shell flash and transformed into ${ }^{22} \mathrm{Ne}$. In non-HBB AGB stars a neutron released from the ${ }^{22} \mathrm{Ne}(\alpha, \mathrm{n})^{25} \mathrm{Mg}$ reaction can be captured by ${ }^{22} \mathrm{Ne}$ again, leading to the production of ${ }^{23} \mathrm{Na}$; this ${ }^{22} \mathrm{Ne} n$-capture is very efficient. In more massive AGB stars, the dominant effect is the Ne-Na cycle burning of dredged-up ${ }^{22} \mathrm{Ne}$ during HBB. In any case, the predicted AGB production range is $[\mathrm{Na} / \mathrm{Fe}] \sim+1.0$ to $\sim+1.7$. Again, since this is a primary production, this number would to first order increase at lower $[\mathrm{Fe} / \mathrm{H}]$.

Hence, while rotating massive stars have been shown to be able to mix primary $\mathrm{C}$ out of the core into the H-burning shell and thus produce primary $\mathrm{N}, \mathrm{Na}$ production requires that this $\mathrm{N}$ be exposed to He-burning once again. This may be difficult to achieve in massive stars.

Sodium is very low in CS 31080-095, which poses a problem if one interprets its CNO abundance as a result of AGB pollution. The low Na abundance in CS 31080-095, together with its high $\mathrm{Mg}$ and low $\mathrm{Al}$ abundance, does in fact fit the wellknown odd-even pattern that is characteristic of massive-star yields at low metallicity. The other two stars show this oddeven pattern starting at $\mathrm{Mg}$; apparently, some additional primary source of $\mathrm{Na}$ filled in the low $\mathrm{Na}$ expected from the standard odd-even effect.

\subsection{Neutron-capture elements}

The first s-process-peak elements $\mathrm{Sr}$ and $\mathrm{Y}$ are low in our stars ( $\mathrm{Y}$ is detected only in CS 31080-095), at the level seen in

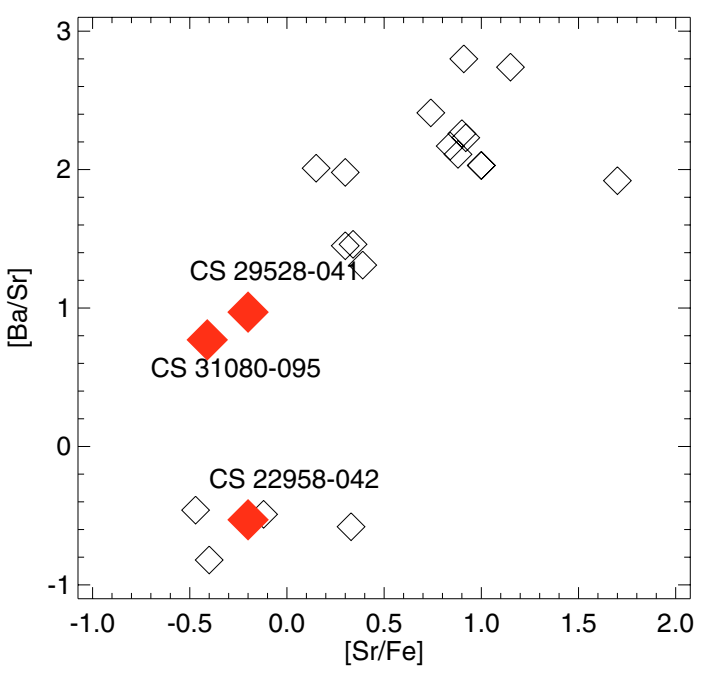

Fig. 5. $[\mathrm{Sr} / \mathrm{Fe}]$ vs. $[\mathrm{Ba} / \mathrm{Sr}]$ for the CEMP stars in Table 4 (filled symbols: this paper). [Ba/Sr] ratios for CS 31080-095 and CS 29528-041 falls in between CEMP-s stars and CEMP-no stars.

field giants of similar metallicity (François et al. 2006). The second-peak s-process element $\mathrm{Ba}$ is moderately overabundant in CS 31080-095 and CS 29528-041; these two stars defy a clear classification into either CEMP-s or CEMP-no, as can be seen in Fig. 5. All the CEMP-no stars listed in Table 4 are mildly C-rich and have much lower Ba than our two stars. At very low metallicities there might be an r-process component of $\mathrm{Ba}$, but $\mathrm{Sr}$ should then be higher than observed in our program stars. Accurate heavy-element abundances for a larger number of CEMP stars is clearly needed to determine the origin of the heavy elements.

In Table 4, CS 31080-095 and CS 29528-041 define a new class, CEMP-no/s, with properties intermediate between the CEMP-no and CEMP-s classes. In contrast, CS 22949-037 (Depagne et al. 2002) displays a moderate Sr enhancement and low Ba abundance.

CS 31080-095 and CS 22958-042 exhibit exactly opposing behaviour with regard to $\mathrm{Ba}$ and $\mathrm{Na}$. The interpretation of this result is not clear. The $\mathrm{C} / \mathrm{N}$ ratio of both stars indicate that they carry the signature of non-HBB AGB stars of low mass. Models predict qualitatively that for non-HBB AGB stars $\mathrm{Ba}$ and $\mathrm{Na}$ should both be enhanced. In particular the large $\mathrm{Na}$ and small Ba abundance of CS 22958-042 means most of the $\mathrm{Na}$ has not been produced by neutron captures on ${ }^{22} \mathrm{Ne}$ in the He-shell flash. However, as explained above, neutron captures are the source $\mathrm{Na}$ in low-mass EMP stellar models. Compared to CS 31080-095 CS 22958-042 has also a larger N/O abundance than the low-mass stellar evolution models (cf. Fig. 6). This indicates that the excess $\mathrm{Na}$ in CS 22958-042 has orinated in p-captures, possibly in a cool-bottom burning type extra- mixing. It is conceivable that tidal interactions in the binary system have induced a variation of mixing, that could explain the difference between CS 31080-095 and CS 22958-042.

\subsection{Li in CEMP stars}

Few CEMP stars presently have spectra of sufficient quality from which abundances or upper limits on $A(\mathrm{Li})$ can be termined. For CEMP-s stars the source of this Li is most likely a former AGB companion star, now a white dwarf. While evolving through the giant phase, low- and intermediate-mass stars 

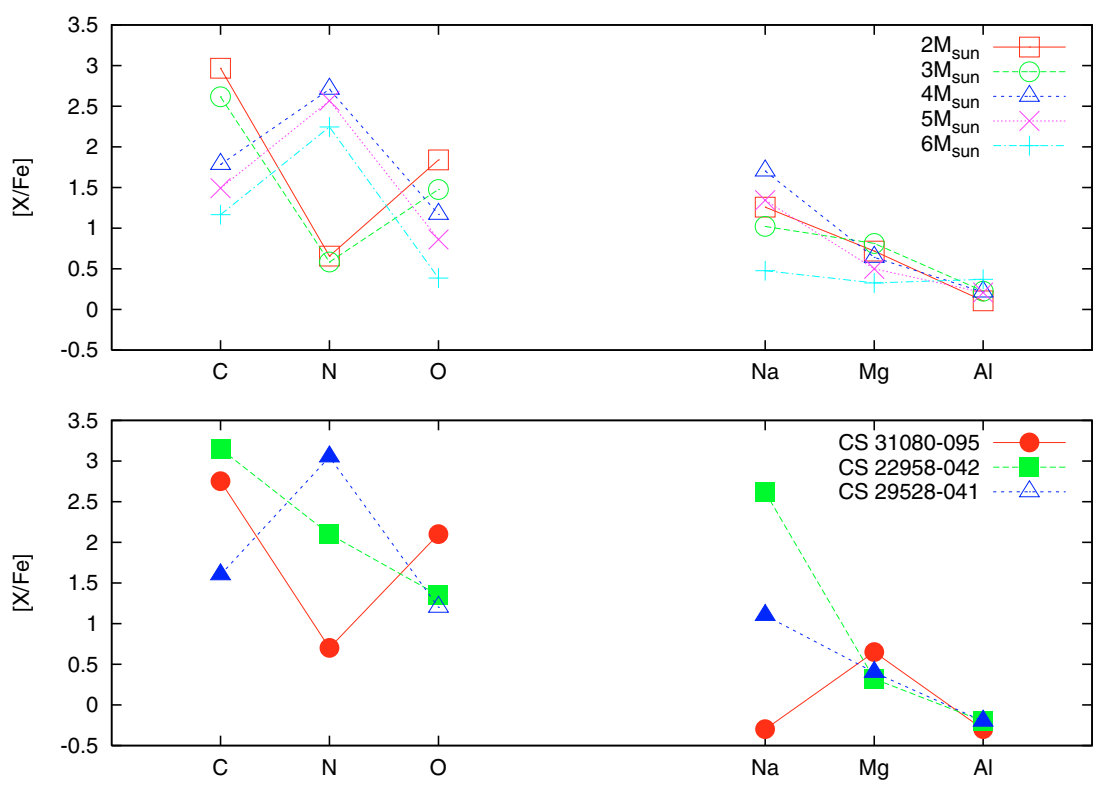

Fig. 6. Top: element enhancements in VMP AGB model ejecta $([\mathrm{Fe} / \mathrm{H}]=-2.3 ; Z=0.0001)$. Bottom: observed abundances in our stars (open symbols: upper limits). The abundances of CS 29528-041 agree well with HBB model predictions for an AGB mass of 4-6 $M_{\odot}$, but the other two stars are better matched by lower-mass AGB models. However, the observed high $\mathrm{N}$ abundances require enhanced mixing, and the observed $\mathrm{Na}$ may differ more than expected from current models. are expected to deplete their initial Li (e.g. CS 22948-027, CS 29497-034, and LP 625-44). Cool dwarfs should also exhibit low $\mathrm{Li}$, due to convection-driven depletion in the star itself (e.g., G 77-61).

Lithium should be preserved in warm dwarfs $\left(T_{\text {eff }}>\right.$ $5700 \mathrm{~K})$. The existence of stars with $A(\mathrm{Li})$ slightly below the Spite Plateau (e.g., CS 31080-095 and CS 29528-041) indicates that some $\mathrm{Li}$ has been destroyed due to mixing processes in the progenitor(s) that produced the $\mathrm{C}$ (and $\mathrm{N}$ ) enhancement. Some other CEMP stars, e.g., CS 22958-042 or CS 29497030 , have values of $T_{\text {eff }}$ and $\log g$ such that Li should not have been depleted; yet $A(\mathrm{Li})$ is considerably below the Spite Plateau. While stars with Li slightly below the Spite Plateau can be explained by transfer of Li-depleted material from an AGB companion, a star like CS 22958-042 must have been Li-poor already before this event.

Finally, the two CEMP-s stars LP 706-7 and CS 22898-027 have $\mathrm{Li}$ abundances corresponding to the Spite Plateau. This implies that the donor star has produced some $\mathrm{Li}$, in an amount finely tuned to keep Li close to the Spite Plateau value on the companion star we now observe. A similar process has been suggested for the main-sequence turnoff stars in the globular cluster NGC 6397 (Bonifacio et al. 2002).

Several mechanisms can in fact produce Li both during the Red Giant Branch (RGB) and AGB phases. On the RGB, a mechanism invoking enhanced, rotationally-induced mixing has been proposed and explored by Denissenkov \& Herwig (2004). This is a particularly effective variant of the cool-bottom processing proposed to explain some of the abundance anomalies in corundum grains and globular clusters (Wasserburg et al. 1995; Boothroyd \& Sackmann 1999).

Enhanced mixing on the RGB can produce Li in a star that has depleted all its primordial Li, but it cannot produce the large overabundances of primary $\mathrm{C}, \mathrm{N}$, and $\mathrm{O}$ that are observed in the CEMP stars. However, if enhanced mixing is possible, and observed in RGB stars, it may be possible on the AGB as well, but it has not been yet been considered in any evolution model for VMP AGB stars. The only quantitative treatment of such an evolution scenario was by Nollett et al. (2003), who focused on isotopic ratios in solar metallicity AGB stars, but not on Li. We may, nevertheless, speculate that dwarf CEMP-s stars with detectable
Li may be the result of enhanced mixing during the AGB phase of their companions.

We cannot exclude this origin for $\mathrm{Li}$ in CS 31080-095. The fact that enhanced-mixing RGB models predict a low ${ }^{12} \mathrm{C} /{ }^{13} \mathrm{C}$ ratio does not necessarily disagree with the high ${ }^{12} \mathrm{C} /{ }^{13} \mathrm{C}$ measured in CS 31080-095, because the enhanced mixing may occur only in a transitional phase, followed (or preceded) by efficient third dredge-up of primary ${ }^{12} \mathrm{C}$. Third dredge-up will increase the ${ }^{12} \mathrm{C} /{ }^{13} \mathrm{C}$ ratio, but in low-mass AGB stars Li could still survive, at least partially. Clearly, a detailed theoretical investigation of this scenario is highly desirable.

Another mechanism to produce $\mathrm{Li}$ in AGB stars is the well-known HBB process, which operates in more massive AGB stars. The lower mass limit for HBB decreases with declining metallicity and is about $3.5 M_{\odot}$ for $[\mathrm{Fe} / \mathrm{H}]=-2.3 . \mathrm{Li}$ production by $\mathrm{HBB}$ and efficient transformation of primary $\mathrm{C}$ into $\mathrm{N}$ are robust predictions of intermediate-mass stellar evolution models; CS 29528-041 seems to fit this pattern.

Finally, a number of studies of the evolution of EMP, UMP, or zero-metallicity AGB stars have invoked the so-called $\mathrm{H}$-ingestion flash to account for the observed abundance patterns in some of the more extreme CEMP stars (Fujimoto et al. 2000; Herwig 2003; Iwamoto et al. 2004; Suda et al. 2004). The $\mathrm{H}$-ingestion flash can also be a source of $\mathrm{Li}$, as was first described by Herwig \& Langer (2001) in the context of the bornagain post-AGB stars (Werner \& Herwig 2006), and confirmed by Iwamoto et al. (2004) for VMP stars and by Suda et al. (2004) for EMP and HMP AGB stars.

Unfortunately, quantitative abundance predictions for this event remain very uncertain because of our poor understanding of the coupled mixing and burning processes that are involved. Probably, a H-ingestion flash that produces $\mathrm{Li}$ is followed by several thermal pulses with efficient ${ }^{12} \mathrm{C}$ dredge-up, which would also make this scenario a candidate for explaining the observed Li abundance in CS 31080-095.

\section{6. ${ }^{12} \mathrm{C} /{ }^{13} \mathrm{C}$ ratios}

Most CEMP-s stars exhibit carbon isotope ratios in the range ${ }^{12} \mathrm{C} /{ }^{13} \mathrm{C} \sim 10-20$, but the CEMP-no stars, including CS 22958-042, have ${ }^{12} \mathrm{C} /{ }^{13} \mathrm{C} \sim 3-10$ (CS 29502-092 is an exception). Figure 7 shows ${ }^{12} \mathrm{C} /{ }^{13} \mathrm{C}$ vs. $A(\mathrm{Li})$ for CEMP stars with 


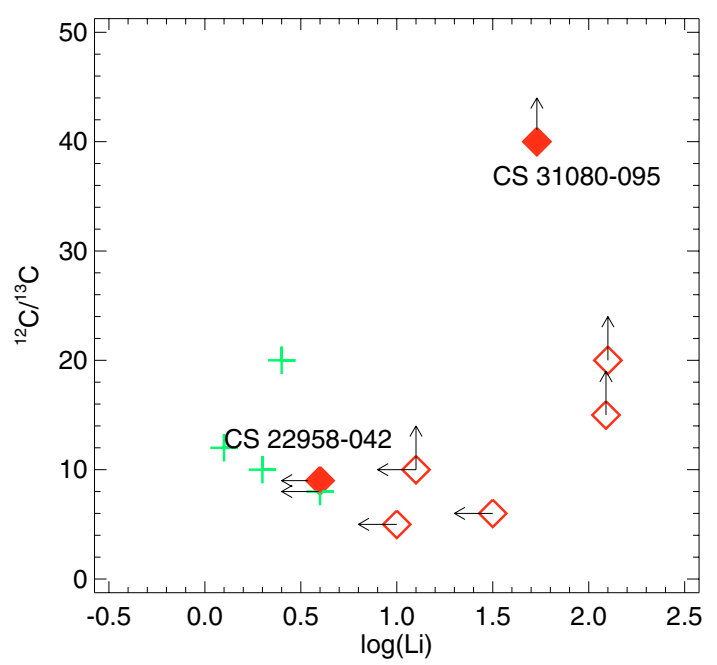

Fig. 7. ${ }^{12} \mathrm{C} /{ }^{13} \mathrm{C}$ vs. $A(\mathrm{Li})$ for the CEMP stars in Table 4 (symbols as in Fig. 4; arrows indicate limits). The Li-rich CEMP stars tend to have high ${ }^{12} \mathrm{C} /{ }^{13} \mathrm{C}$ ratios.

published detections or limits on these two quantities. ${ }^{12} \mathrm{C} /{ }^{13} \mathrm{C}$ appears to be higher the in CEMP stars with detected lithium, but additional efforts to place tighter constraints on both ${ }^{12} \mathrm{C} /{ }^{13} \mathrm{C}$ and $\mathrm{Li}$ in CEMP stars are clearly needed.

Standard VMP AGB star models predict that $\mathrm{HBB}$ produces ${ }^{12} \mathrm{C} /{ }^{13} \mathrm{C} \sim 10$, while non-HBB models yield ${ }^{12} \mathrm{C} /{ }^{13} \mathrm{C}$ of the order of $10^{3}-10^{4}$. The fact that no CEMP star in Table 4 (in particular no CEMP-s or CEMP-r/s star) has such a high ${ }^{12} \mathrm{C} /{ }^{13} \mathrm{C}$ ratio is probably the clearest evidence that standard AGB models are neglecting some mixing mechanism. Even at solar metallicities the AGB models yield higher ${ }^{12} \mathrm{C} /{ }^{13} \mathrm{C}$ ratios than are observed, indicating the need for extra mixing (Charbonnel 1998). The extramixing process invoked above to account for the unexpectedly low $\mathrm{C} / \mathrm{N}$ ratios in all CEMP-s stars is qualitatively suitable to produce the observed ${ }^{12} \mathrm{C} /{ }^{13} \mathrm{C}$ ratios as well.

\section{Conclusions}

The origin of the observed abundance patterns of CEMP stars remains enigmatic. Figure 6 summarises how the abundances in our stars compare to the predicted abundances in AGB ejecta of very low metallicity, presumably transferred to the observed stars from former AGB binary companions. It should be recognized that the stars have lower $[\mathrm{Fe} / \mathrm{H}]$ than the models. Because all the overabundances shown are dominated by the primary production in AGB stars, this could be corrected to first order by just adding the $\mathrm{Fe}$ abundance difference (in logarithmic units). However, a stringent comparison of models and actual stellar observations must include the problems of mass transfer and dilution. For that reason, one should not pay too much attention to absolute overabundances of $0.5-1$ dex, but rather focus on the abundance patterns.

In our small sample, CS $29528-041$ is probably the most straightforward case; all available evidence points toward pollution from a HBB AGB star. CS 29528-041 is one of the very few known NEMP stars, the existence of which is predicted by standard stellar evolution in conjunction with the binary mass-transfer scenario for CEMP-s stars. The moderate Ba overabundance in this star may be due to a polluting AGB star of large mass. The CNO abundances would also be compatible with pollution from a massive, rotating star. The s-process in these (or indeed any VMP AGB) stars is not yet understood.
However, the observed $\mathrm{Li}, \mathrm{Na}$, and $\mathrm{Ba}$ abundances clearly favour the AGB alternative.

Assuming that the measured rapid velocity variation for CS 22958-042 is due to a binary orbital motion of short period, the AGB connection strongly suggests that this star is a member of a post-common-envelope binary. This must be the case if the overabundances are attributed to mass-transfer from an AGB star.

We can only speculate about the effects of common-envelope phase on the nuclear signatures in a CEMP star that formed from this mechanism. One scenario could involve several thermal pulses with efficient dredge-up causing the observed overabundances of $\mathrm{C}, \mathrm{N}, \mathrm{O}$ and $\mathrm{Na}$. However, before the s-process production can get fully underway, AGB evolution is terminated by the onset of common-envelope evolution. This could explain the absence of $\mathrm{Ba}$ in this star.

Another CEMP star with a short orbital period is HE 00242523 (Lucatello et al. 2003), which must be a post-common envelope object as well. However, in that star Ba (and other heavy elements) are overabundant without doubt. These two shortperiod binaries have contrasting $\mathrm{Na}$ signatures: $\mathrm{Na}$ is underabundant in HE 0024-2523, but CS 22958-042 is one of the most Na-rich stars yet found.

Both HE 0024-2523 and CS 22958-042 have very large $\mathrm{C} / \mathrm{O}$ ratios, although $\mathrm{O}$ is significantly overabundant: $\mathrm{C} / \mathrm{O}=48$ in CS 22958-042, and 100 in HE 0024-2523. A very large $\mathrm{C} / \mathrm{O}$ ratio may present a problem for the AGB mass-transfer scenario, because a non-HBB AGB star can only deliver material with a lower $\mathrm{C} / \mathrm{O}$ ratio than found anywhere inside of it. The material dredged up to the surface, usually from the intershell, contains from one to a few percent primary $\mathrm{O}$ by mass, almost independent of metallicity, and also maybe $20-25 \%$ carbon by mass ${ }^{1}$. Thus, $\mathrm{C} / \mathrm{O}<32$ is a reasonable limit for the intershell layers of AGB stars (including VMP AGB stars). Even if the AGB star dumps this maximally polluted material onto the CEMP star we now observe, the resulting $\mathrm{C} / \mathrm{O}$ ratio cannot become larger than in the intershell layer. The $\mathrm{C} / \mathrm{O}$ ratio could be a problem for the two known short-period binaries, but perhaps especially for HE 0024-2523.

Clearly, common-envelope evolution introduces a whole new family of possible evolutionary outcomes. Nevertheless, it is noteworthy that we may have found the second short-period CEMP binary, which seems to be significantly different from the previously known case, HE 0024-2523. One of the two might in fact not be a post-common envelope system, but have an inert low-mass MS companion instead. However, VMP AGB stars remain the best present candidate nuclear production sites for explaining both the s-process enhancement in HE 0024-2523 and the high $\mathrm{Na}$ in CS 22958-042.

Finally, CS 31080-095 could be an AGB mass-transfer object if VMP non-HBB AGB stars experience some extra mixing. This could account for the observed $\mathrm{Li}, \mathrm{C}$, and $\mathrm{N}$ abundances. The $\mathrm{O}$ abundance in this star is certainly high compared to the predictions of this scenario, but cannot rule it out. The problem is the low $\mathrm{Na}$ abundance, which excludes AGB progenitors above $2 M_{\odot}$. Perhaps a less massive AGB star is involved, in which the ${ }^{22} \mathrm{Ne}$ source is not activated at all; the Ba could then still come from the ${ }^{13} \mathrm{C}$ neutron source. The larger ${ }^{12} \mathrm{C} /{ }^{13} \mathrm{C}$ ratio $(>40)$ in this star is consistent with this picture, and inconsistent with the obvious alternatives; the ${ }^{12} \mathrm{C} /{ }^{13} \mathrm{C}$ ratios in $\mathrm{HBB}$

\footnotetext{
1 These $\mathrm{C}$ and $\mathrm{O}$ abundances are for models without extra convective mixing. If such mixing is included both $\mathrm{C}$ and $\mathrm{O}$ increase, but the $\mathrm{C} / \mathrm{O}$ ratio does not change much.
} 
AGB stars $\left({ }^{12} \mathrm{C} /{ }^{13} \mathrm{C} \sim 10\right)$ as well as the wind ejecta of rotating massive stars $\left({ }^{12} \mathrm{C} /{ }^{13} \mathrm{C} \sim 4\right)$ are much lower than the observed limit.

This discussion has clearly shown that our current understanding of the origin of the abundance patterns in CEMP stars is still in its infancy. The large overabundances of some elements in CEMP stars probe one or more important primary nuclear production sites, including those that may have set the stage for the following galactic chemical evolution. Yet, we remain far from having identified even the main players in this game. Tasks to be addressed include a better understanding of mixing in stars of all masses, and of the impact of binary evolution on nucleosynthesis. A significant increase in the numbers of CEMP stars with accurate, detailed elemental abundances is also an obvious next step.

Acknowledgements. We thank the referee for the comments and suggestions, which has considerably improved the paper. We thank the ESO staff for assistance during all the runs of our Large Programme. R.C., P.F., V.H., B.P., F.S. and M.S. thank the PNPS and the PNG for their support. P.B. and P.M. acknowledge support from the MIUR/PRIN 2004025729_002 and P.B. from EU contract MEXT-CT-2004-014265 (CIFIST). T.C.B. and T.S. acknowledge partial funding for this work from grants AST 00-98508, AST 00-98549, and AST 04-06784, as well as from grant PHY 02-16783: Physics Frontiers Center/Joint Institute for Nuclear Astrophysics (JINA), all from the U.S. National Science Foundation. B.N. and J.A. thank the Carlsberg Foundation and the Swedish and Danish Natural Science Research Councils for partial financial support of this work. F.H. acknowledges support from the LDRD program at LANL through grant 20060357ER.

\section{References}

Alonso, A., Arribas, S., \& Martínez-Roger, C. 1996, A\&A, 313, 873 Alvarez, R., \& Plez, B. 1998, A\&A, 330, 1109

Aoki, W., Norris, J. E., Ryan, S. G., Beers, T. C., \& Ando, H. 2000, ApJ, 536, L97

Aoki, W., Ryan, S. G., Norris, J. E., et al. 2001, ApJ, 561, 346

Aoki, W., Norris, J. E., Ryan, S. G., Beers, T. C., \& Ando, H. 2002a, ApJ, 576, L141

Aoki, W., Norris, J. E., Ryan, S. G., Beers, T. C., \& Ando, H. 2002b, ApJ, 567, 1166

Aoki, W., Ryan, S. G., Norris, J. E., et al. 2002c, ApJ, 580, 1149

Aoki, W., Ryan, S. G., Tsangarides, S., et al. 2003, IAUJD, 15, 19

Aoki, W., Norris, J. E., Ryan, S. G., et al. 2004, ApJ, 608, 971

Aoki, W., Beers, T. C., Christlieb, N., et al. 2006a, ApJ, submitted

Aoki, W., Frebel, A., Christlieb, N., et al. 2006b, ApJ, 639, 897

Asplund, M. 2005, ARA\&A, 43, 481

Asplund, M., Grevesse, N., \& Sauval, A. J. 2005, Cosmic Abundances as Records of Stellar Evolution and Nucleosynthesis, ASP Conf. Ser., 336, 25 Barbuy, B., Spite, M., Spite, F., et al. 2005, A\&A, 429, 1031

Barklem, P. S., Christlieb, N., Beers, T. C., et al. 2005, A\&A, 439, 129

Baumüller, D., \& Gehren, T. 1997, A\&A 325, 1088

Baumüller, D., Butler, K., \& Gehren, T. 1998, A\&A 338, 637

Beers, T. C. 1999, The Third Stromlo Symposium: The Galactic Halo, ASP Conf. Ser., 165, 202

Beers, T. C., \& Christlieb, N. 2005, ARA\&A, 43, 531

Beers, T. C., Preston, G. W., \& Shectman, S. A. 1985, AJ, 90, 2089

Beers, T. C., Preston, G. W., \& Shectman, S. A. 1992, AJ, 103, 1987

Bonifacio, P., Molaro, P., Beers, T. C., \& Vladilo, G. 1998, A\&A, 332, 672

Bonifacio, P., Pasquini, L., Spite, F., et al. 2002, A\&A, 390, 91

Bonifacio, P., Limongi, M., \& Chieffi, A. 2003, Nature, 422, 834

Boothroyd, A. I., \& Sackmann, I.-J. 1999, ApJ, 510, 232

Cayrel, R., Depagne, E., Spite, M., et al. 2004, A\&A 416, 1117 ("First Stars V")

Charbonnel, C. 1998, Abundance Profiles: Diagnostic Tools for Galaxy History, ASP Conf. Ser., 147, 157

Chiappini, C., Hirschi, R., Meynet, G., et al. 2006, A\&A, 449, L27

Christlieb, N. 2003, Rev. Mod. Astron., 16, ed. R. Schielicke (Berlin: WileyVCH), 191

Christlieb, N., Bessell, M. S., Beers, T. C., et al. 2002, Nature, 419, 904

Cohen, J. G., Christlieb, N., Qian, Y.-Z., \& Wasserburg, G. J. 2003, ApJ, 588, 1082
Dekker, H., D’Odorico, S., Kaufer, A., Delabre, B., \& Kotzlowski, H. 2000, Proc. SPIE, 4008, 534

Denissenkov, P. A., \& Herwig, F. 2004, ApJ, 612, 1081

Depagne, E., Hill, V., Spite, M., et al. 2002, A\&A, 390, 187 ("First Stars II")

François, P., Depagne, E., Hill, V., et al. 2003, A\&A, 403, 1105 ("First Stars III")

François, P., Depagne, E., Hill, V., et al. 2006, A\&A, in prep. ("First Stars VIII")

Frebel, A., Aoki, W., Christlieb, N., et al. 2005, Nature, 434, 871

Frebel, A., Christlieb, N., Norris, J. E., Aoki, W., \& Asplund, M. 2006, ApJ, 638, L17

Fujimoto, M. Y., Ikeda, Y., \& Iben, I., Jr. 2000, ApJ, 529, L25

Gratton, R. G., Carretta, E., Eriksson, K., \& Gustafsson, B. 1999, A\&A, 350, 955

Gustafsson, B., Edvardsson, B., Eriksson, K., et al. 2003, IAU Symp., 210, 4P

Herwig, F., \& Langer, N. 2001, Nucl. Phys. A, 688, 221

Herwig, F. 2003, CNO in the Universe, ASP Conf. Ser., 304, 318

Herwig, F. 2004, ApJS, 155, 651

Herwig, F. 2005, ARA\&A, 43, 435

Hill, V., Barbuy, B., Spite, M., et al. 2000, A\&A, 353, 557

Hirschi, R., Fröhlich, C., Liebendörfer, M., \& Thielemann, F.-K. 2006, in Rev. Mod. Astron. 19, in press [arXiv:astro-ph/0601502]

Ivans, I. I., Sneden, C., Gallino, R., Cowan, J. J., \& Preston, G. W. 2005, ApJ, 627, L145

Iwamoto, N., Kajino, T., Mathews, G. J., Fujimoto, M. Y., \& Aoki, W. 2004, ApJ, 602, 377

Iwamoto, N., Umeda, H., Tominaga, N., Nomoto, K., \& Maeda, K. 2005, Science, 309, 451

Johnson, J. A., Herwig, F., Beers, T. C., \& Christlieb, N. 2005, Nucl. Phys. A, 758,221

Johnson, J. A., Herwig, F., Beers, T. C., \& Christlieb, N. 2006, ApJ, in press

Jonsell, K., Barklem, P. S., Gustafsson, B., et al. 2006, A\&A, 451, 651

Karlsson, T. 2006, ApJ, in press [arXiv:astro-ph/0602597]

Kinman, T., \& Castelli, F. 2002, A\&A, 391, 1039

Kiselman, D. 1991, A\&A, 245, L9

Kiselman, D. 2001, New Astron. Rev., 45, 559

Kupka, F., Piskunov, N. E., Ryabchikova, T. A., Stempels, H. C., \& Weiss, W. W. 1999, A\&AS, 138, 119

Kurucz, R. L. 1993, CD-ROM, 15

Lucatello, S., Gratton, R., Cohen, J. G., et al. 2003, AJ, 125, 875

Lucatello, S., Tsangarides, S., Beers, T. C., et al. 2005, ApJ, 625, 825

Lucatello, S., Beers, T. C., Christlieb, N., et al. 2006, ApJ, submitted

McWilliam, A. 1998, AJ, 115, 1640

Meynet, G., Ekström, S., \& Maeder, A. 2006, A\&A, 447, 623

Nollett, K. M., Busso, M., \& Wasserburg, G. J. 2003, ApJ, 582, 1036

Norris, J. E., Ryan, S. G., Beers, T. C., \& Deliyannis, C. P. 1997a, ApJ, 485, 370

Norris, J. E., Ryan, S. G., \& Beers, T. C. 1997b, ApJ, 488, 350

Norris, J. E., Ryan, S. G., \& Beers, T. C. 1997c, ApJ, 489, L169

Norris, J. E., Ryan, S. G., \& Beers, T. C. 1999, ApJS, 123, 639

Norris, J. E., Ryan, S. G., Beers, T. C., Aoki, W., \& Ando, H. 2002, ApJ, 569, L107

Piau, L., Beers, T. C., Balsara, D. S., et al. 2006, ApJ, submitted [arXiv: astro-ph/0603553]

Plez, B., \& Cohen, J. G. 2005, A\&A, 434, 1117

Plez, B., Cohen, J. G., \& Meléndez, J. 2005, IAU Symp., 228, 267

Preston, G. W., \& Sneden, C. 2001, AJ, 122, 1545

Ryan, S. G., Aoki, W., Norris, J. E., \& Beers, T. C. 2005, ApJ, 635, 349

Sackmann, I.-J., \& Boothroyd, A. I. 1999, ApJ, 510, 217

Schlegel, D. J., Finkbeiner, D. P., \& Davis, M. 1998, ApJ, 500, 525

Schuster, W. J., Michel, R., \& Beers, T. C. 2005, Rev. Mex. Astron. Astrofis. Conf. Ser., 24, 273

Sivarani, T., Bonifacio, P., Molaro, P., et al. 2004, A\&A, 413, 1073 ("First Stars IV")

Skrutskie, M. F., Cutri, R. M., Stiening, R., et al. 2006, AJ, 131, 1163

Sneden, C., Cowan, J. J., Lawler, J. E., et al. 2003, ApJ, 591, 936

Spite, F., \& Spite, M. 1986, A\&A, 163, 140

Spite, M., Cayrel, R., Plez, B., et al. 2005, A\&A, 430, 655 ("First Stars VI")

Spite, M., Cayrel, R., Hill, V., et al. 2006, A\&A, 455, 291 ("First Stars IX")

Suda, T., Aikawa, M., Machida, M. N., Fujimoto, M. Y., \& Iben, I. Jr. 2004, ApJ, 611,476

Takeda, Y. 1994, PASJ, 46, 53

Thorburn, J. A. 1994, ApJ, 421, 318

Thorburn, J. A., \& Beers, T. C. 1992, BAAS, 24, 1278

Tsangarides, S., Ryan, S. G., \& Beers, T. C. 2004, Mem. Soc. Astron. Ital., 75, 772

Van Eck, S., Goriely, S., Jorissen, A., \& Plez, B. 2003, A\&A, 404, 291

Wasserburg, G. J., Boothroyd, A. I., \& Sackmann, I.-J. 1995, ApJ, 447, L37

Werner, K., \& Herwig, F. 2006, PASP, in press [arXiv : astro-ph/0512320] 